\title{
Specific-heat discontinuity in impure two-band superconductors
}

\author{
Todor M. Mishonov,,$^{1,2, *}$ Evgeni S. Penev, ${ }^{2}$ Joseph O. Indekeu, ${ }^{1, \dagger}$ and Valery L. Pokrovsky ${ }^{3, \dagger}$ \\ ${ }^{1}$ Laboratorium voor Vaste-Stoffysica en Magnetisme, Katholieke Universiteit Leuven, Celestijnenlaan 200 D, B-3001 Leuven, Belgium \\ ${ }^{2}$ Faculty of Physics, Sofia University "St. Kliment Ohridski,” 5 James Bourchier Boulevard, 1164 Sofia, Bulgaria \\ ${ }^{3}$ Department of Physics, Texas A\&M University, College Station, Texas 77843-4242, USA \\ and Landau Institute for Theoretical Physics, Kosygin Street 2, Moscow 117940, Russia
}

(Received 14 September 2002; revised manuscript received 17 March 2003; published 18 September 2003)

\begin{abstract}
The Ginzburg-Landau coefficients and the jump of the specific heat are calculated for a disordered two-band superconductor. We start with the analysis of a more general case with arbitrary anisotropy. While the specificheat discontinuity at the critical temperature $T_{c}$ decreases with increasing disorder, its ratio to the normal-state specific heat at $T_{c}$ increases and slowly converges to the isotropic value. For strong disorder the deviation from the isotropic value is proportional to the elastic electron-scattering time. In the case of a two-band superconductor we apply a simplified model of the interaction independent of the momentum within a band. In the framework of this model all thermodynamic variables can be found explicitly at any value of the scattering rate. This solution explains the sample dependence of the specific-heat discontinuity in $\mathrm{MgB}_{2}$ and the influence of disorder on the critical temperature.
\end{abstract}

DOI: 10.1103/PhysRevB.68.104517

PACS number(s): 74.20.Fg, 74.72.-h

\section{INTRODUCTION}

The investigation of the specific heat $C(T)$ is an important tool for understanding the nature of the superconductivity and the anisotropy of the superconducting gap $\Delta_{\mathbf{p}}(T)$ on the Fermi surface $\varepsilon_{\mathrm{p}}=E_{\mathrm{F}}$. Historically, the relative specificheat jump $\Delta C / C_{n}\left(T_{c}\right)$ was used to establish the BCS picture $^{1}$ for conventional superconductors having nearly isotropic gap. Subsequently the thermodynamics of clean anisotropic-gap superconductors was analyzed in the weakcoupling approximation by Pokrovskii and Ryvkin., ${ }^{2,3}$ They have found that anisotropy suppresses the value $\Delta C / C_{n}\left(T_{c}\right)$ in comparison to its isotropic value 1.43 . This inequality is not satisfied in classical low-temperature superconductors partly because they are not extremely clean, but also since the weak-coupling approximation has a poor precision. Geilikman and Kresin ${ }^{4}$ have proved that the first correction due to interaction increases $\Delta C / C_{n}\left(T_{c}\right)$ and thus disguises the effect of anisotropy. The modern superconductors display considerable anisotropy. In particular, superconductivity is highly anisotropic in $\mathrm{MgB}_{2}$. This fact is the main motivation for this work. It is well known that only superconducting crystals of very high quality can reach the theoretical cleanlimit asymptotics. As a rule, the reduced specific-heat jump is sample dependent, and understanding such a disorder dependence is a challenging problem. The latter is especially important for $\mathrm{MgB}_{2}$, a compound now being in the limelight of superconductor materials science.

The aim of the present paper is to derive the dependence of the critical temperature and the relative specific-heat jump $\Delta C / C_{n}\left(T_{c}\right)$ on the elastic-scattering time of the charge carriers at the critical temperature $\tau\left(T_{c}\right)$ for two-band superconductors having in mind application to $\mathrm{MgB}_{2}$. For this purpose we need corresponding formulas for a general dirty anisotropic superconductor. Such equations were derived in Ref. 5. We reproduce them here for the reader's convenience and because several misprints occurred in the cited work which we correct here. In the $\tau$-approximation the electrical resistivity of the normal metal $\rho_{\mathrm{el}}$ is determined by this scattering time. Thus, our formula can be used for the investigation of correlations in the experimentally determined $\Delta C / C_{n}\left(T_{c}\right)$ versus $\rho_{\mathrm{el}}\left(T_{c}\right) / T_{c}$ plot. The comparison of the theoretical curve and the experimental data can reveal the gap anisotropy $\Delta_{b, \mathbf{p}}$ and the scattering rate $1 / \tau\left(T_{c}\right)$. The gap may depend on both the quasimomentum $\mathbf{p}$ and the band index $b$.

The applicability of the weak-coupling theory to $\mathrm{MgB}_{2}$ is controversial. However, experimental results on the relative specific-heat discontinuity ${ }^{6}$ indicate that the anisotropy effect is more profound than the effect of interaction. For this compound, the reduced specific heat $\Delta C / C_{n}\left(T_{c}\right)$ is definitely smaller than the weak-coupling BCS value 1.43. Moreover, the temperature dependence of the specific heat of the superconducting phase is described fairly well ${ }^{7}$ by the two-band model $^{8}$ and the relative specific-heat jump agrees ${ }^{7}$ with the Moskalenko weak-coupling formula. ${ }^{8}$ The comparison of the latter with the $a b$ initio strong-coupling calculations ${ }^{9}$ for $\mathrm{MgB}_{2}$ shows that the decrease of $\Delta C / C_{n}\left(T_{c}\right)$, due to different values of the superconducting gap for different bands, is at least two times bigger than the increase of this reduced specific heat-jump due to strong-coupling effects. We address this point in the concluding section.

\section{CLEAN SUPERCONDUCTORS}

In this section we reproduce some results for anisotropic clean superconductors obtained by different authors many years ago and derive an equation for the specific heat discontinuity in this case. Though none of these results are new, they are necessary for understanding the next sections.

It was shown in Ref. 3 that, within the framework of the weak-coupling theory, the order parameter possesses the property of separability:

$$
\Delta_{\mathbf{p}}(T)=\Xi(T) \chi_{\mathbf{p}}
$$


According to Eq. (1), the temperature dependence characterized by the factor $\Xi(T)$ is separated from the angular dependence described by the factor $\chi_{\mathbf{p}}$. The Ginzburg-Landau (GL) expansion for the free-energy density ${ }^{10-12}$ can be written in terms of the temperature-dependent factor $\Xi(T)$ alone:

$$
f(\Xi, T)=a_{0} \frac{T-T_{c}}{T_{c}}|\Xi|^{2}+\frac{1}{2} b|\Xi|^{4} .
$$

The specific-heat jump per unit volume is related to the GL coefficients by the following relation:

$$
\left.\left(C_{s}-C_{n}\right)\right|_{T_{c}}=\Delta C=\frac{1}{T_{c}} \frac{a_{0}^{2}}{b},
$$

where $C_{s}$ is the specific heat per unit volume of the superconducting phase and $C_{n}$ is that of the normal phase.

Our starting points are the expressions of Gor'kov and Melik-Barkhudarov ${ }^{13}$ for the GL coefficients in the clean limit which can be written as

$$
a_{0}=\nu_{\mathrm{F}}\left\langle\chi^{2}\right\rangle, \quad b=\frac{\zeta\left(3, \frac{1}{2}\right) \nu_{\mathrm{F}}}{2\left(2 \pi k_{\mathrm{B}} T_{c}\right)^{2}}\left\langle\chi^{4}\right\rangle,
$$

where the Hurwitz and the Riemann zeta functions, $\zeta(k, z)$ and $\zeta(k)$, respectively, read

$$
\zeta(k, z)=\sum_{n=0}^{\infty}(n+z)^{-k}, \quad \zeta(k)=\zeta(k, 1)=\sum_{n=1}^{\infty} n^{-k},
$$

and obey the relation $\zeta\left(k, \frac{1}{2}\right)=\left(2^{k}-1\right) \zeta(k)$. A simple variational derivation of Eq. (4) is given in Ref. 14. The celebrity of $\zeta(3)$ in mathematics has been discussed in Ref. 15. The normalized moments of the gap-anisotropy function are determined by averaging over the Fermi surface, having the general form in the $D$-dimensional case

$$
\begin{aligned}
\left\langle\chi^{n}\right\rangle & \equiv \int \cdots \int_{\mathrm{BZ}} \chi_{\mathbf{p}}^{n} \delta\left(\varepsilon_{\mathbf{p}}-E_{\mathrm{F}}\right) \frac{d \mathbf{p}}{\nu_{\mathrm{F}}(2 \pi \hbar)^{D}} \\
& =\int \cdots \int_{\varepsilon_{\mathbf{p}}=E_{\mathrm{F}}} \chi_{\mathbf{p}^{n}}^{n} \frac{d S_{\mathbf{p}}}{\nu_{\mathrm{F}} v_{\mathbf{p}}(2 \pi \hbar)^{D}},
\end{aligned}
$$

where $d S_{\mathbf{p}}$ is an infinitesimal surface element and $\mathbf{v}_{\mathbf{p}}=\nabla_{\mathbf{p}} \varepsilon_{\mathbf{p}}$ is the quasiparticle velocity. The quasimomentum space integral is taken over the whole Brillouin zone (BZ). The integration over the Fermi surface $\varepsilon_{\mathbf{p}}=E_{\mathrm{F}}$ implicitly includes summation over fragments and sheets of different bands, if any. The normalizing factor

$$
\nu_{\mathrm{F}}=\int \cdots \int_{\varepsilon_{\mathbf{p}}=E_{\mathrm{F}}} \frac{d S_{\mathbf{p}}}{v_{\mathbf{p}}(2 \pi \hbar)^{D}}
$$

is the density of states (DOS) per unit volume for fixed spin, and enters the normal-phase specific heat

$$
C_{n}(T)=\frac{2}{3} \pi^{2} k_{\mathrm{B}}^{2} \nu_{\mathrm{F}} T
$$

This equation together with the formulas for the GL coefficients, Eq. (4), leads to the following expression for the reduced jump of the specific heat:

$$
\begin{gathered}
\frac{\Delta C}{C_{n}\left(T_{c}\right)}=\frac{12}{7 \zeta(3)} \frac{1}{\beta_{\Delta}}, \\
\frac{1}{\beta_{\Delta}}=\frac{\left\langle\Delta_{\mathbf{p}}^{2}\right\rangle^{2}}{\left\langle\Delta_{\mathbf{p}}^{4}\right\rangle}=\frac{\left\langle\chi^{2}\right\rangle^{2}}{\left\langle\chi^{4}\right\rangle} \leqslant 1, \\
\frac{12}{7 \zeta(3)}=1.42613 \ldots,
\end{gathered}
$$

which is exactly the result obtained in Refs. 2 and 3; for a methodical derivation see Ref. 14. Using Eq. (4) for $T$ slightly lower than $T_{c}$, we get for the equilibrium order parameter

$$
\begin{gathered}
|\Xi|_{\mathrm{eq}}^{2}=-\frac{T-T_{c}}{T_{c}} \frac{a_{0}}{b}, \\
\left|\Delta_{\mathbf{p}}\right|^{2}=|\Xi|_{\mathrm{eq}}^{2} \chi_{\mathbf{p}}^{2}=\frac{2\left(2 \pi k_{\mathrm{B}} T_{c}\right)^{2}}{\zeta\left(3, \frac{1}{2}\right)} \frac{T_{c}-T}{T_{c}} \frac{\left\langle\chi^{2}\right\rangle}{\left\langle\chi^{4}\right\rangle} \chi_{\mathbf{p}}^{2},
\end{gathered}
$$

which is the result by Gor'kov and Melik-Barkhudarov. ${ }^{13}$

\section{DISORDERED ANISOTROPIC SUPERCONDUCTORS}

\section{A. Transition line and order parameter}

In this section we analyze the transition temperature $T_{c}$ as a function of the elastic-scattering rate $1 / \tau$ and the angular dependence of the order parameter $\chi_{\mathbf{p}}$. As was explained before, the angular dependence is the same for any temperature at fixed $\tau$. The transition line has been studied in Ref. 5 . Although the equations obtained in the latter work were rather general, their treatment was focused on a specific situation - a mixture of $s$ - and $d$-pairing characteristic for cuprate superconductors. Therefore, it is useful to analyze the results for a less exotic case of anisotropic s-pairing. The general equation for the transition line found in Ref. 5 reads

$$
g\left(T_{c}, \tau\right) \sum_{n} \frac{V_{n}\left|\left\langle\Psi_{n}\right\rangle\right|^{2}}{1-f\left(T_{c}, \tau\right) V_{n}}=1,
$$

where the following notations are introduced:

$$
\begin{gathered}
f(T, \tau)=\frac{1}{\pi}\left[\ln \frac{\bar{\epsilon}}{2 \pi k_{\mathrm{B}} T}-\digamma\left(x+\frac{1}{2}\right)\right], \\
g(T, \tau)=\frac{1}{\pi}\left[\digamma\left(x+\frac{1}{2}\right)-\digamma\left(\frac{1}{2}\right)\right],
\end{gathered}
$$

$x=\left(2 \pi k_{\mathrm{B}} T \tau / \hbar\right)^{-1} ; \bar{\epsilon}$ is the cutoff energy; $\digamma(x)$ is the Euler digamma function; $V_{n}$ are eigenvalues of the linear operator $\hat{V}$ with kernel $V\left(\mathbf{p}, \mathbf{p}^{\prime}\right)$ equal to the electron-electron effective interaction energy at the Fermi surface multiplied by the $\operatorname{DOS} \nu_{\mathrm{F}} ; \Psi_{n}(\mathbf{p})$ are the corresponding eigenfunctions nor- 
malized according to the condition $\left\langle\left|\Psi_{n}\right|^{2}\right\rangle=1$. The transition temperature of the clean superconductor is determined by the equation $f\left(T_{c 0}, \tau=\infty\right)=V_{0}^{-1}$ which gives

$$
k_{\mathrm{B}} T_{c 0}=\frac{2 \gamma \bar{\epsilon}}{\pi} \exp \left(-\pi / V_{0}\right), \quad \chi \equiv \Psi_{0},
$$

where

$$
\gamma=\exp C=\frac{1}{4 \exp [\digamma(1 / 2)]}=1.781,
$$

$C=0.577$ is the Euler constant, and $V_{0}$ is the maximum eigenvalue of the operator $\hat{V}$. The angular dependence of the order parameter in this case is given by the corresponding eigenfunction $\Psi_{0}(\mathbf{p})$. As long as $x_{0}=\left(2 \pi k_{\mathrm{B}} T_{c 0} \tau / \hbar\right)^{-1}$ remains large the transition temperature $T_{c}$ differs from $T_{c 0}$ by insignificant corrections of the order of $x_{0}^{-1}$. We call the superconductor moderately dirty if the value $x_{0}$ becomes small, but $f\left(T_{c}, \tau\right)$ is still close to $V_{0}^{-1}$. More precisely, it means that $\left|\ln (\bar{\epsilon} \tau / \hbar)-\pi V_{0}^{-1}\right| \ll V_{0}^{-1}$. Then the solution of Eq. (11) reads

$$
T_{c}(\tau)=T_{c 0}\left(\frac{\tau}{\tau_{0}}\right)^{\kappa-1}
$$

where $\kappa=\left\langle\chi^{2}\right\rangle /\langle\chi\rangle^{2} \geqslant 1$ is the anisotropy coefficient, which is equal to 1 for an isotropic superconductor, and $\tau_{0}$ $=2 \hbar \gamma /\left(\pi k_{\mathrm{B}} T_{c 0}\right)$. In this range of the scattering rate the angular dependence of the gap $\chi_{\mathbf{p}}$ is identical to that for the clean superconductor: $\chi_{\mathbf{p}}=\Psi_{0}(\mathbf{p})$. Thus, the transition temperature decreases in a powerlike way with the increase of the scattering rate $1 / \tau$ or the residual resistivity $\rho_{\text {res }}$ proportional to this rate. This is a peculiarity of the anisotropic superconductor. The exponent in Eq. (16) is zero for the isotropic superconductor, cf. Ref. 16. Equation (16) was first derived by Hohenberg ${ }^{17}$ for weakly anisotropic superconductors. Its validity for arbitrary $\kappa$ in the range of moderate dirt was proven in Ref. 5.

We call the dirt strong if the parameter $\ln (\bar{\epsilon} \tau / \hbar) / \pi$ becomes less than $V_{0}^{-1}$ and has the order of magnitude of $V_{0}^{-1}$, and if the difference $V_{0}^{-1}-\ln (\bar{\epsilon} \tau / \hbar) / \pi$ is not small in comparison to $V_{0}^{-1}$. Equation (16) remains qualitatively correct, but $\kappa$ becomes a slowly varying function of $\tau$. The exact formula for the transition temperature in this range is given by Eq. (37) of Ref. 5.

In the extra-dirty limit, $\ln (\bar{\epsilon} \pi / \hbar) / \pi$ becomes much smaller than $V_{0}^{-1}$, but still $\bar{\epsilon} \tau / \hbar \gg 1$. The last inequality ensures that the elastic scattering does not destroy the Fermi surface. In the extra-dirty limit the angular dependence of the gap reaches its limiting value $\chi_{\mathbf{p}} \propto \bar{V}(\mathbf{p})$, where $\bar{V}(\mathbf{p})$ $=\left\langle V\left(\mathbf{p}, \mathbf{p}^{\prime}\right)\right\rangle_{\mathbf{p}^{\prime}}$. The equation for $T_{c}$ in the extra-dirty limit reads

$$
k_{\mathrm{B}} T_{c}(\tau)=\frac{2 \gamma \bar{\epsilon}}{\pi} \exp \left(-\frac{\pi}{\langle\bar{V}\rangle}\right)(\bar{\epsilon} \tau / \hbar)^{\bar{\kappa}-1},
$$

where $\langle\bar{V}\rangle=\langle\bar{V}(\mathbf{p})\rangle_{\mathbf{p}}$. It should be noted that $\bar{\kappa}$ in the last equation differs from that for the clean superconductor, namely,

$$
\bar{\kappa}=\frac{\sum_{n} V_{n}^{2}\left\langle\Psi_{n}\right\rangle^{2}}{\left(\sum_{n} V_{n}\left\langle\Psi_{n}\right\rangle\right)^{2}} .
$$

\section{B. Specific-heat discontinuity}

The theory of dirty anisotropic superconductors ${ }^{5}$ was based on Green's functions method combined with the Abrikosov-Gor'kov averaging over the random impurity field. ${ }^{18,19}$ A simplifying assumption was the isotropy of the scattering which is characterized by a constant rate $1 / \tau$. In particular, the authors derived the GL equations and GL coefficients with an accuracy of a common scaling factor ${ }^{5}$ (see also Ref. 20). For the representation adopted here this factor is $\left\langle\chi^{2}\right\rangle$, as it follows from the comparison of Eq. (4) here and Eqs. (48), (60), (78)-(82) in Ref. 5. Correcting a misprint in Eq. (59) in Ref. 5, further repeated in Eqs. (61) and (82) therein, and slightly regrouping terms we find

$$
\begin{gathered}
a_{0}=\nu_{\mathrm{F}}\left[\left\langle\chi^{2}\right\rangle-\left(\left\langle\chi^{2}\right\rangle-\langle\chi\rangle^{2}\right) x_{c} \zeta_{2,0}\right] \\
b=\frac{\nu_{\mathrm{F}}}{8\left(\pi k_{\mathrm{B}} T_{\mathrm{c}}\right)}\left[\left\langle\chi^{4}\right\rangle \zeta_{3,0}-\left\langle\chi^{2}\right\rangle^{2} x_{c} \zeta_{4,0}+4\left\langle\chi^{3}\right\rangle\langle\chi\rangle x_{c} \zeta_{3,1}\right. \\
\left.+2\left\langle\chi^{2}\right\rangle\langle\chi\rangle^{2}\left(x_{c}^{3} \zeta_{4,2}+x_{c}^{2} \zeta_{3,2}\right)+\langle\chi\rangle^{4} x_{c}^{4} \zeta_{4,3}\right]
\end{gathered}
$$

where

$$
x_{c}=\frac{\hbar / \tau\left(T_{c}\right)}{2 \pi k_{\mathrm{B}} T_{c}}=\frac{x_{0}}{T_{c} / T_{c 0}}
$$

is the dimensionless scattering rate extrapolated to the critical temperature. The resistivity of the normal metal $\rho_{\mathrm{el}}$ is determined by the Drude formula:

$$
\rho_{\mathrm{el}}^{-1}=m^{-1} n e^{2} \tau,
$$

where $m$ is the effective mass and $n$ is the density of normal charge carriers; for clean crystals the total volume density of all charge carriers, electrons and holes, $e n=e\left(n_{e}-n_{h}\right)$, can be determined by the Hall constant $\mathcal{R}_{\mathrm{H}}=1 /$ en in strong magnetic fields. For clean superconductors, disregarding some subtleties, the same ratio $\mathrm{n} / \mathrm{m}$ enters the London penetration depth $\lambda_{\text {clean }}$ at $T=0$ :

$$
\frac{1}{\lambda_{\text {clean }}^{2}(0)}=\frac{n e^{2}}{m c^{2} \varepsilon_{0}},
$$

where in Gaussian units $\varepsilon_{0}=1 / 4 \pi ; \lambda_{\text {clean }}(0) \sim 0.1-1 \mu \mathrm{m}$. Multiplying these equations we obtain a useful estimate

$$
x_{c} \simeq \frac{\hbar c^{2} \varepsilon_{0} \rho_{\mathrm{el}}\left(T_{c}\right)}{2 \pi \lambda_{\text {clean }}^{2}(0) k_{\mathrm{B}} T_{c}} .
$$


The notation $\zeta_{k, l}$ in Eq. (20) stands for the generalized $\zeta$ functions defined in Ref. 5 and taken at the value of the argument ${ }^{21} z_{c}=x_{c}+1 / 2$, i.e.,

$$
\zeta_{k, l} \equiv \zeta_{k, l}\left(z_{c}\right)
$$

For the reader's convenience we recall the definition of these functions:

$$
\zeta_{k, l}(z)=\sum_{n=0}^{\infty}(n+z)^{-k}(n+1 / 2)^{-l} .
$$

They represent a natural generalization of the Hurwitz zeta functions:

$$
\zeta_{k, 0}(z)=\zeta(k, z), \quad \zeta_{k, 0}(1)=\zeta(k, 1)=\zeta(k) .
$$

Below we provide the asymptotics of $\zeta_{k, l}(z)$ for $z \rightarrow \infty$ necessary for the further calculations:

$$
\begin{gathered}
\zeta_{k, l}(z) \sim\left(2^{l}-1\right) \zeta(l) z^{-k}-k\left[\left(2^{l-1}-1\right) \zeta(l-1)\right. \\
\left.-\left(2^{l}-1\right) \zeta(l) / 2\right] z^{-k-1} \text { for } l>2, \\
\zeta_{k, l}(z) \sim\left(2^{l}-1\right) \zeta(l) z^{-k} \text { for } \quad l=2, \\
\zeta_{k, 1}(z) \sim z^{-k} \ln z \text { for } \quad k \geqslant 1, \\
\zeta_{k, 0}(z) \sim(k-1)^{-1} z^{-k+1}+\frac{1}{2} z^{-k} \text { for } k>1 .
\end{gathered}
$$

Let us note that, for integer arguments $k$, the Hurwitz zeta functions are associated with the Euler polygamma function $\digamma^{(k)}$ :

$$
\zeta(k+1, z)=\frac{(-1)^{k+1}}{k !} \digamma^{(k)}(z), \quad k=1,2,3, \ldots
$$

With these notations the reduced discontinuity of the specific heat reads

$$
\frac{\Delta C}{C_{n}\left(T_{c}\right)}=\frac{a_{0}^{2}}{k_{\mathrm{B}} T_{c} b}=\frac{12}{7 \zeta(3)} \frac{1}{\beta_{\tau}},
$$

where

$$
\begin{aligned}
\frac{1}{\beta_{\tau}}= & 7 \zeta(3)\left[\left\langle\chi^{2}\right\rangle-\left(\left\langle\chi^{2}\right\rangle-\langle\chi\rangle^{2}\right) x_{c} \zeta_{2,0}\right]^{2} \\
& \times\left[\left\langle\chi^{4}\right\rangle \zeta_{3,0}-\left\langle\chi^{2}\right\rangle^{2} x_{c} \zeta_{4,0}+4\left\langle\chi^{3}\right\rangle\langle\chi\rangle x_{c} \zeta_{3,1}\right. \\
& \left.+2\left\langle\chi^{2}\right\rangle\langle\chi\rangle^{2}\left(x_{c}^{3} \zeta_{4,2}+x_{c}^{2} \zeta_{3,2}\right)+\langle\chi\rangle^{4} x_{c}^{4} \zeta_{4,3}\right]^{-1} .
\end{aligned}
$$

This general equation will be applied in the following sections to some important special cases.

It should be stressed that for isotropic superconductors, $\left\langle\chi^{n}\right\rangle=1$, the specific-heat jump is impurity independent: $\beta_{\tau}=1$. The proof is straightforward taking into account the identity

$$
\begin{aligned}
& \zeta_{3,0}-x_{c} \zeta_{4,0}+4 x_{c} \zeta_{3,1}+2\left(x_{c}^{3} \zeta_{4,2}+x_{c}^{2} \zeta_{3,2}\right)+x_{c}^{4} \zeta_{4,3} \\
& =\zeta(3,1 / 2)=7 \zeta(3) .
\end{aligned}
$$

Likewise, using Eq. (28) one can prove that the asymptotic form of $\Delta C / C_{n}\left(T_{c}\right)$ for an extremely disordered superconductor with an arbitrary anisotropy is given by, to leading order in $x_{c}^{-1}$,

$$
\frac{\Delta C}{C_{n}\left(T_{c}\right)} \sim \frac{12}{7 \zeta(3)}\left[1-\frac{2 \pi^{2}}{7 \zeta(3)} \frac{\kappa-1}{x_{c}}\right] .
$$

We remark that the correction of order $1 / x_{c}$ comes entirely from the coefficient $b$ given in Eq. (20).

\section{Two-band superconductors}

\section{Critical curve and order parameter}

Keeping in mind the application to $\mathrm{MgB}_{2}$ (for a review see Ref. 22), we apply the general results of the previous sections to a simplified model of a two-band superconductor. In this model we assume that the Fermi surface consists of two disconnected sheets having different DOS. The interaction amplitude $V\left(\mathbf{p}, \mathbf{p}^{\prime}\right)$ is assumed to be a constant within each band. Thus, it can be described by a $2 \times 2$ matrix

$$
\hat{V}=\left(\begin{array}{cc}
W_{1} & U \\
U & W_{2}
\end{array}\right),
$$

where $W_{1}, W_{2}$ are the interaction energies between any two points within the first and the second sheet of the Fermi surface, respectively; $U$ is the interaction between any two points of different bands.

Let us first work out the transition temperature $T_{c 0}$ and the order parameter $\chi$ for a clean two-band superconductor. ${ }^{8}$ For our simplified model the order parameter $\chi_{\mathbf{p}}$ is a constant within each band, i.e., it can be represented by a twocomponent vector

$$
\chi=\left(\begin{array}{l}
\chi_{1} \\
\chi_{2}
\end{array}\right) .
$$

The eigenvectors $\Psi$ of the operator $\hat{V}$ obey the following linear equations:

$$
\begin{aligned}
& c_{1} W_{1} \Psi_{1}+c_{2} U \Psi_{2}=\lambda \Psi_{1}, \\
& c_{1} U \Psi_{1}+c_{2} W_{2} \Psi_{2}=\lambda \Psi_{2},
\end{aligned}
$$

where the coefficients $c_{1,2}=\nu_{1,2} /\left(\nu_{1}+\nu_{2}\right)$ are the statistical weights of the two bands, which reflect the integral character of the operator $\hat{V}$. The two independent eigenvalues of Eqs. (35) read

$$
V_{0,1} \equiv \lambda_{ \pm}=\eta \pm \epsilon
$$

where

$$
\begin{gathered}
\eta=\frac{1}{2}\left(c_{1} W_{1}+c_{2} W_{2}\right), \quad \epsilon=\sqrt{\xi^{2}+c_{1} c_{2} U^{2}}, \\
\xi=\frac{1}{2}\left(c_{1} W_{1}-c_{2} W_{2}\right) .
\end{gathered}
$$

The corresponding eigenvectors are 


$$
\begin{gathered}
\Psi_{+}=\left(\begin{array}{c}
\sqrt{\frac{1}{2 c_{1}}(1+\xi / \epsilon)} \\
\operatorname{sgn}(U) \sqrt{\frac{1}{2 c_{2}}(1-\xi / \epsilon)}
\end{array}\right), \\
\Psi_{-}=\left(\begin{array}{c}
-\operatorname{sgn}(U) \sqrt{\frac{1}{2 c_{1}}(1-\xi / \epsilon)} \\
\sqrt{\frac{1}{2 c_{2}}(1+\xi / \epsilon)}
\end{array}\right) .
\end{gathered}
$$

This is apparently a kind of Bogolyubov transformation. Both vectors are normalized:

$$
\left\langle\left|\Psi_{ \pm}\right|^{2}\right\rangle=c_{1}\left|\Psi_{ \pm, 1}\right|^{2}+c_{2}\left|\Psi_{ \pm, 2}\right|^{2}=1 .
$$

The average values of the anisotropic eigenfunctions read

$$
\begin{gathered}
\left\langle\Psi_{+}\right\rangle=\sqrt{\frac{c_{1}}{2}\left(1+\frac{\xi}{\epsilon}\right)}+\operatorname{sgn}(U) \sqrt{\frac{c_{2}}{2}\left(1-\frac{\xi}{\epsilon}\right)}, \\
\left\langle\Psi_{-}\right\rangle=-\operatorname{sgn}(U) \sqrt{\frac{c_{1}}{2}\left(1-\frac{\xi}{\epsilon}\right)}+\sqrt{\frac{c_{2}}{2}\left(1+\frac{\xi}{\epsilon}\right)} .
\end{gathered}
$$

It is useful to write simple expressions for the squared averages:

$$
\begin{gathered}
\left\langle\Psi_{ \pm}\right\rangle^{2}=\frac{1}{2} \pm \frac{1}{2 \epsilon}\left[\left(c_{1}-c_{2}\right) \xi+2 c_{1} c_{2} U\right], \\
\left\langle\Psi_{+}\right\rangle^{2}+\left\langle\Psi_{-}\right\rangle^{2}=1, \quad\langle\chi\rangle^{2}=\left\langle\Psi_{+}\right\rangle^{2} .
\end{gathered}
$$

For the gap ratio $\delta$, Eq. (38) gives

$$
\delta \equiv \frac{\Delta_{1}}{\Delta_{2}}=\frac{\Psi+, 1}{\Psi_{+, 2}}=\frac{\chi_{1}}{\chi_{2}}=\operatorname{sgn}(U) \sqrt{\frac{c_{2}}{c_{1}}} \sqrt{\frac{\epsilon+\xi}{\epsilon-\xi}} .
$$

Whence the moments of the anisotropy function read

$$
\left\langle\chi^{n}\right\rangle=\frac{\left\langle\Delta^{n}\right\rangle}{\left\langle\Delta^{2}\right\rangle^{n / 2}}=\frac{c_{1} \delta^{n}+c_{2}}{\left(c_{1} \delta^{2}+c_{2}\right)^{n / 2}} .
$$

Using the general results formulated earlier, we find the transition temperature of the clean two-band superconductor:

$$
T_{c 0}=\frac{2 \gamma \bar{\epsilon}}{\pi} \exp \left(-\pi / \lambda_{+}\right)
$$

Equation (11) for the critical curve within this model can be simplified to the following form:

$$
\begin{aligned}
\pi g[ & \left.\eta+\left(c_{1}-c_{2}\right) \xi+2 c_{1} c_{2} U-c_{1} c_{2} f d\right] \\
& =\left(1-\lambda_{+} f\right)\left(1-\lambda_{-} f\right) .
\end{aligned}
$$

Here we have denoted $d=\operatorname{det} \hat{V}=W_{1} W_{2}-U^{2}$, and abbreviated the functions $f\left(T_{c}, \tau\right)$ and $g\left(T_{c}, \tau\right)$ as $f$ and $g$, respectively, cf. Eqs. (12) and (13). It is convenient to recall the dimensionless scattering rate

$$
x_{0}=\left(2 \pi k_{\mathrm{B}} T_{c 0} \tau / \hbar\right)^{-1},
$$

and the dimensionless transition temperature $\theta$ $=T_{c}(\tau) / T_{c 0} ; x_{c}=x_{0} / \theta$. In terms of these variables the functions $f$ and $g$ read

$$
\begin{gathered}
f=\frac{1}{\lambda_{+}}-\frac{1}{\pi} \ln \theta-g, \\
\pi g=\digamma\left(x_{c}+\frac{1}{2}\right)-\digamma\left(\frac{1}{2}\right) .
\end{gathered}
$$

The equation for $T_{c}$ finally takes the form

$$
g\left[\langle\chi\rangle^{2} \lambda_{+}+\left(1-\langle\chi\rangle^{2}\right) \lambda_{-}-f \lambda_{+} \lambda_{-}\right]=\left(1-f \lambda_{+}\right)\left(1-f \lambda_{-}\right),
$$

where

$$
\langle\chi\rangle^{2}=\frac{\left(c_{1} \delta+c_{2}\right)^{2}}{c_{1} \delta^{2}+c_{2}}, \quad c_{2}=1-c_{1} .
$$

To illustrate the possible dependence $\theta\left(x_{0}\right)$, we have made a numerical calculation setting ${ }^{9} \delta=2.63, \lambda_{+}=1.02, \lambda_{-}$ $=0.45$, and $c_{1}=0.422$; other authors calculate slightly different values for the two-band model ${ }^{8,23,24}$ applied to $\mathrm{MgB}_{2}$, cf. Refs. 6,7, and 25-27. The results for $\theta\left(x_{0}\right)$ and $\Delta C / C_{n}\left(T_{c}\right)$ versus $x_{c}$ are shown in Fig. 1.

In the asymptotic regions of moderate and extreme dirt, Eq. (16) is valid with

$$
\kappa=\left\{\frac{1}{2}\left[1+\frac{\left(c_{1}-c_{2}\right) \xi}{\epsilon}\right]+\frac{c_{1} c_{2} U}{\epsilon}\right\}^{-1}=\frac{c_{1} \delta^{2}+c_{2}}{\left(c_{1} \delta+c_{2}\right)^{2}}
$$

for the clean and moderate dirt cases, and

$$
\bar{\kappa}=\frac{c_{1}^{3} W_{1}^{2}+c_{2}^{3} W_{2}^{2}+c_{1} c_{2}(2 \eta+U) U}{\left(c_{1}^{2} W_{1}+c_{2}^{2} W_{2}+2 c_{1} c_{2} U\right)^{2}}
$$

for the extreme dirt case. The order parameter in the moderate dirt range is proportional to $\Psi_{+}$. In the range of strong disorder it reads

$$
\chi=\left(\begin{array}{c}
c_{1} W_{1}+c_{2} U-\pi^{-1} c_{1} c_{2} d \ln (\bar{\epsilon} \tau / \hbar) \\
c_{1} U+c_{2} W_{2}-\pi^{-1} c_{1} c_{2} d \ln (\bar{\epsilon} \tau / \hbar)
\end{array}\right) .
$$

In the limit of extreme disorder, $\ln (\bar{\epsilon} \tau / \hbar) \ll W_{1,2}^{-1}$, it tends to the limiting value:

$$
\chi_{\text {extr }}=\bar{V}=\left(\begin{array}{l}
c_{1} W_{1}+c_{2} U \\
c_{1} U+c_{2} W_{2}
\end{array}\right)
$$

It is worth noting that at $W_{1}=W_{2}=U$ the anisotropy parameter $\kappa$ is equal to 1 for any value of the scattering rate independent of the values $c_{1}, c_{2}$, and all thermodynamical values are independent of $\tau$ similar to the completely isotropic case.

\section{Specific-heat discontinuity}

We note that the normalization factors of the gapanisotropy function and the superconducting gap mutually cancel in the formulas for the experimentally measurable jump of the specific heat. Therefore, we can use the normal- 

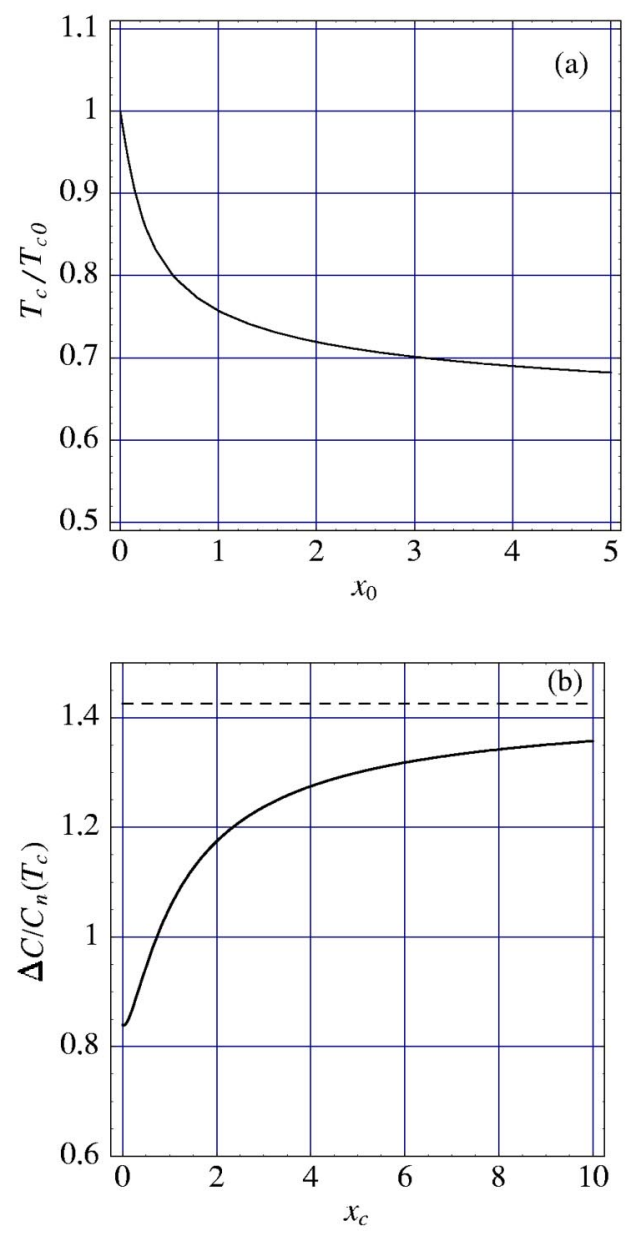

FIG. 1. (a) Critical curve $T_{c}(\tau)$ for the two-band model: the reduced critical temperature $\theta=T_{c}(\tau) / T_{c 0}$ vs dimensionless scattering rate $x_{0}=\hbar / 2 \pi k_{\mathrm{B}} T_{c 0} \tau \propto \rho_{\mathrm{el}}\left(T_{c}\right)$. The set of parameters corresponds to $\mathrm{MgB}_{2}$; for details see the text. (b) Reduced specific-heat jump $\Delta C / C_{n}\left(T_{c}\right)$ as a function of the dimensionless scattering rate $x_{c}=\hbar / 2 \pi k_{\mathrm{B}} T_{c} \tau \propto \rho_{\mathrm{el}}\left(T_{c}\right) / T_{c}$ for the same set of parameters. The dashed line indicates the asymptotic (BCS) value for $x_{c} \gg 1$. The curve will be shifted up by about $20 \%$ due to strong-coupling effects.

ization $\left\langle\chi^{2}\right\rangle=1$ without loss of generality. The calculations made above can be used directly to find the averages necessary for the calculation of the specific heat. The influence of the disorder on the relative specific-heat discontinuity for a two-band superconductor is shown in Fig. 1(b). In the extreme dirty limit we find that the relative specific-heat discontinuity tends to its isotropic value in agreement with the fact that the density of states becomes isotropic in this limit. ${ }^{5}$

\section{Dirty isotropic alloys}

It is straightforward to verify that in the isotropic case ( $\chi=$ const on the Fermi surface) the coefficients $a_{0}$ and $b$ do not depend on $\tau$. Thus, neither the energy gap nor the specific heat is influenced by impurities, in accordance with the Anderson theorem. ${ }^{16}$ Analyzing Eq. (11), we conclude that the transition temperature also does not depend on the scattering rate. Indeed, in the isotropic case the eigenfunctions of the operator $\hat{V}$ are spherical harmonics $\Psi_{l m}=Y_{l m}(\theta, \varphi)$. Among the latter only one, $\Psi_{00}$, has a nonzero average. Thus, Eq. (11) takes the simple form

$$
g\left(T_{c}, \tau\right)+f\left(T_{c}, \tau\right)=V_{0}^{-1} .
$$

According to definitions (12) and (13), the sum $f(T, \tau)$ $+g(T, \tau)=\pi^{-1}\left[\ln \left(\bar{\epsilon} / 2 \pi k_{\mathrm{B}} T\right)-\digamma(1 / 2)\right]$ does not depend on the scattering rate. Hence, $T_{c}$ also does not depend on the scattering rate.

\section{E. Separable approximation}

The separable approximation

$$
V\left(\mathbf{p}, \mathbf{p}^{\prime}\right)=\sum_{n} V_{n} \Psi_{n}^{*}(\mathbf{p}) \Psi_{n}\left(\mathbf{p}^{\prime}\right) \approx V_{0} \chi_{\mathbf{p}} \chi_{\mathbf{p}^{\prime}},
$$

where $\Psi_{0}(\mathbf{p}) \equiv \chi_{\mathbf{p}}$, is very often used for modeling the gap anisotropy in superconductors $\Delta_{\mathbf{p}} \approx \Xi(T) \chi_{\mathbf{p}}$. As we demonstrated earlier, this approximation is valid in the range of clean and moderately dirty superconductors. Applying this approximation to the equation for $T_{c}$ in the two-band model, Eq. (49), we obtain the Moskalenko and Palistrant, ${ }^{24}$ Abrikosov $^{28}$ and Kogan equation ${ }^{29}$

$$
\ln \frac{T_{c 0}}{T_{c}(\tau)}=\left(1-\frac{\left\langle\Delta_{\mathbf{p}}\right\rangle^{2}}{\left\langle\Delta_{\mathbf{p}}^{2}\right\rangle}\right)\left[\digamma\left(x_{c}+\frac{1}{2}\right)-\digamma\left(\frac{1}{2}\right)\right],
$$

where $^{24} 1 / \tau=\frac{1}{2}\left(1 / \tau_{12}+1 / \tau_{21}\right)$, and $1 / \tau_{12}$ and $1 / \tau_{21}$ are rates of interband scattering. The results of the numerical solution of this equation are depicted in Fig. 2. For superconductors with zero averaged gap, $\left\langle\Delta_{\mathbf{p}}\right\rangle=0$, which are $p$ - and $d$-type superconductors, for example, this equation formally coincides with the Abrikosov-Gor'kov result ${ }^{18,30}$ for superconductors with magnetic impurities; superconductivity disappears at the critical value $x_{0}=1 / 4 \gamma=0.1404$. For weak disorder we have

$$
T_{c 0}-T_{c} \approx \frac{\left\langle\chi^{2}\right\rangle-\langle\chi\rangle^{2}}{\left\langle\chi^{2}\right\rangle} \frac{\pi \hbar}{4 k_{\mathrm{B}} \tau} \ll T_{c 0} .
$$

Hence, one of the most important properties of multigap and anisotropic superconductors is that the nonmagnetic impurities are pair breaking, similar to magnetic impurities in conventional superconductors. ${ }^{5,24}$ A similar influence of structural defects was discussed by Abrikosov $^{31}$ for triplet superfluids. The reduction of the critical temperature by disorder has been observed for layered cuprates, ${ }^{32-34,23}$ for impurity scattering in the triplet superconductor ${ }^{35} \mathrm{UPt}_{3}$ and recently for $\mathrm{MgB}_{2}{ }^{22}$ Only dimensionless ratios of the gap function moments, such as $\langle\chi\rangle^{2} /\left\langle\chi^{2}\right\rangle$ in Eq. (55), or $\left\langle\chi^{2}\right\rangle^{2} /\left\langle\chi^{4}\right\rangle$ in Eq. (9), are relevant for the thermodynamics of superconductors. This explains why strongly anisotropicgap layered cuprates were seemingly successfully analyzed as two-band superconductors ${ }^{23}$ (this reference is a comprehensive review of the properties of multigap superconductors), and vice versa why the first prominent two-gap superconductor $\mathrm{MgB}_{2}$ could be analyzed as if it were a singleband anisotropic-gap superconductor; ${ }^{36}$ cf. Ref. 37. 


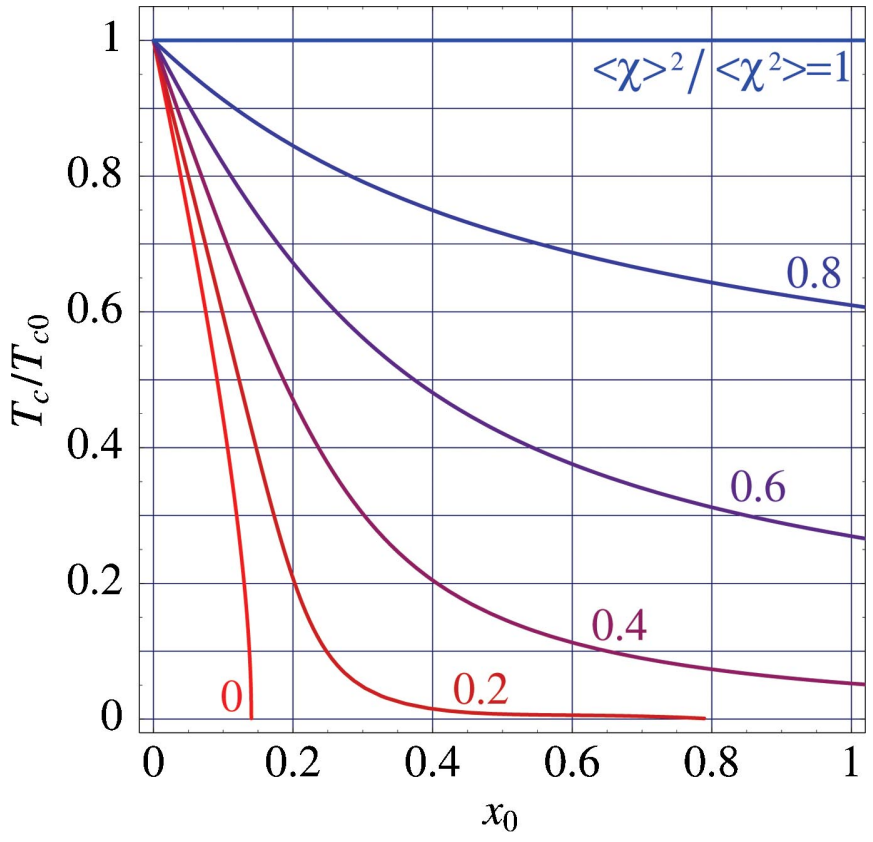

FIG. 2. Critical curves $\theta=T_{c} / T_{c 0}$ vs $x_{0}=\hbar / 2 \pi k_{\mathrm{B}} T_{c 0} \tau$ for different gap anisotropies $\langle\chi\rangle^{2} /\left\langle\chi^{2}\right\rangle=0,0.2,0.4,0.6,0.8$, and 1. For an isotropic superconductor, $\langle\chi\rangle=1$, the critical temperature is disorder independent while for $p$ - or $d$-type superconductors (as some $\mathrm{CuO}_{2}$ superconductors are) $\langle\chi\rangle=0$ and the transition line is exactly the same as the Abrikosov-Gor'kov ${ }^{18,30}$ curve for magnetic impurities in isotropic superconductors.

\section{DISCUSSION AND SUMMARY}

In order to investigate the dependence of $\Delta C / C_{n}\left(T_{c}\right)$ on $1 / \tau T_{c}$ one needs a good method for the determination of $\tau$. This could be far-infrared measurement of the highfrequency conductivity, or merely the static resistivity. For polycrystalline materials one has to use compounds such as $\mathrm{MgB}_{2}$ with good contact between grains. The variation of the residual resistivity can be achieved by radiation.

For $\mathrm{MgB}_{2}$ the gap ratio $\delta=\Delta_{1} / \Delta_{2}$ can be determined by spectroscopic measurements, and the ratio of the DOS $\nu_{1} / \nu_{2}$ can be calculated from first principles. Under these conditions, for high-quality clean samples we can evaluate the up-shift of the $\Delta C / C_{n}\left(T_{c}\right)$ curve, and the influence of strong-coupling effects. Another complication is related to the variation of the electron wave functions in the two bands. This can lead to different scattering rates in the two bands. If the scattering time cannot be determined by spectroscopic measurements one can use the value of the resistivity at the critical temperature $\rho_{\mathrm{el}}\left(T_{c}\right) / T_{c}$. Thus, the dependence of $T_{c}$ and the relative specific-heat jump on resistivity at $T=T_{c}$ will be given by our formula and Figs. 2 and 1(b), with the scale of the abscissa being a fitting parameter. We expect that the derived weak-coupling formula can be as useful for the analysis of $\Delta C$ versus $\rho_{\text {el }}$ correlations as the weak-coupling theory was successful in describing the temperature dependence of the specific heat for the clean $\mathrm{MgB}_{2}$ samples. ${ }^{7}$ Thus, we conclude that the weak-coupling theory of the impurity reduction of the specific-heat jump can reveal the main trend and qualitative properties of the effect.

Here we wish to emphasize that according to the analysis of the two-band mode ${ }^{24}$ only the interband scattering rate $1 / \tau_{\pi \sigma}$ is relevant to the reduction of the transition temperature. However, resistivity is created mainly by intraband scattering $1 / \tau_{\pi}$, cf. Ref. 38. Consequently, proper scaling of the resistivity abscissa is needed in the experimental data of $T_{c} / T_{c 0}$ versus $\rho_{\mathrm{el}}$, and the subsequent data analysis can become an important tool for the determination of $\tau_{\pi \sigma} / \tau_{\pi}$ $\gg 1$. This scattering rate ratio is specific for every type of disorder in $\mathrm{MgB}_{2}$. We conclude that only properly scaled data of $T_{c}$ versus $\rho_{\mathrm{el}}$ should display the universal behavior shown in Fig. 2.

Irradiated superconductors are also a good example for the application of the present theory. Furthermore, let us note that conventional dirty superconducting alloys, for which a big enough series of samples with continuously changing resistivity can be prepared, are the best tool to investigate the influence of disorder on the thermodynamics of superconductors. Finally, let us summarize our results.

(i) In anisotropic superconductors the transition temperature is suppressed by disorder like $T_{c} \sim \tau^{\kappa-1}$, where $\kappa$ $=\left\langle\chi^{2}\right\rangle /\langle\chi\rangle^{2}$ is an anisotropy parameter which is a slowly varying function of $\tau$.

(ii) The order parameter retains its angular dependence as long as $\bar{\epsilon} \tau / \hbar \gg 1$, whereas the DOS becomes isotropic for $\ln (\bar{\epsilon} \tau / \hbar) \ll V_{0}^{-1}$. The anisotropy of the order parameter can be probed in tunneling experiments.

(iii) The specific heat is suppressed by disorder just like $T_{c}$, with an accuracy of a slowly varying factor.

(iv) The relative jump of the specific heat $\Delta C / C_{n}\left(T_{c}\right)$ is smaller than its isotropic value in the clean limit. It is enhanced by disorder tending to its isotropic limit in the extreme disordered case.

(v) In isotropic superconductors, and the two-band model with $\Delta_{1}=\Delta_{2}$ and arbitrary $c_{1} / c_{2}, T_{c}$ and $\Delta C$ do not depend on the scattering rate $1 / \tau$; in particular, this is the case for $W_{1}=W_{2}=U$.

\section{ACKNOWLEDGMENTS}

This work was supported by the Flemish Government Program GOA and IUAP. V.L.P. acknowledges support from NSF under Grant No. DMR0072115 and by the von Humboldt Foundation. He is also thankful to Professor W. Selke and RWTH Aachen for the hospitality.
*Fax: +32-16-327983; email address: todor.mishonov@ fys.kuleuven.ac.be

†Email address: joseph.indekeu@fys.kuleuven.ac.be

Email address: valery@physics.tamu.edu
${ }^{1}$ J. Bardeen, L.N. Cooper, and J.R. Schrieffer, Phys. Rev. 108, 1175 (1957), Eq. (3.46).

${ }^{2}$ V.L. Pokrovskii, Zh. Éksp. Teor. Fiz. 40, 641 (1961) [Sov. Phys. JETP 13, 447 (1961)]. 
${ }^{3}$ V.L. Pokrovskiı̌ and M.S. Ryvkin, Zh. Éksp. Teor. Fiz. 43, 92 (1962) [Sov. Phys. JETP 16, 67 (1963)].

${ }^{4}$ B.T. Geilikman and V.Z. Kresin, Zh. Éksp. Teor. Fiz. Pis'ma 5, 271 (1967) [Sov. Phys. JETP Lett. 5, 220 (1967)].

${ }^{5}$ S.V. Pokrovsky and V.L. Pokrovsky, Phys. Rev. B 54, 13275 (1996).

${ }^{6}$ Y. Wang, T. Plackowski, and A. Junod, Physica C 355, 179 (2001)

${ }^{7}$ F. Bouquet, Y. Wang, R.A. Fisher, D.G. Hinks, J.D. Jorgensen, A. Junod, and N.E. Phillips, Europhys. Lett. 56, 856 (2001).

${ }^{8}$ V.A. Moskalenko, Fiz. Met. Metalloved. 8, 503 (1959) [Phys. Met. Metallogr. 8, 25 (1959)].

${ }^{9}$ A.A. Golubov, J. Kortus, O.V. Dolgov, O. Jepsen, Y. Kong, O.K. Andersen, B.J. Gibson, K. Ahn, and R.K. Kremer, J. Phys.: Condens. Matter 14, 1353 (2002).

${ }^{10}$ L.D. Landau and E.M. Lifshitz, Course on Theoretical Physics, Vol. 5, Statistical Physics, Part 1 (Pergamon Press, Oxford, 1980), Chap. XIV.

${ }^{11}$ V.L. Ginzburg and L.D. Landau, Zh. Éksp. Teor. Fiz. 20, 1064 (1950); English translation in Men of Physics: L. D. Landau, edited by D. ter Haar (Pergamon, New York, 1965), Vol. 1, pp. $138-167$.

${ }^{12}$ E.M. Lifshitz and L. Pitaevskii, Course on Theoretical Physics, Vol. 9, Statistical Physics, Part 2 (Pergamon Press, Oxford, 1981), Secs. 45 and 51.

${ }^{13}$ L.P. Gor'kov and T.K. Melik-Barkhudarov, Zh. Éksp. Teor. Fiz. 45, 1493 (1963) [Sov. Phys. JETP 18, 1031 (1964)].

${ }^{14}$ T. Mishonov and E. Penev, Int. J. Mod. Phys. B 16, 3573 (2002).

${ }^{15}$ J.M. Borwein, D.M. Braedley, and R.E. Crandall, J. Comput. Appl. Math. 121, 247 (2000), Sec. 8.

${ }^{16}$ P.W. Anderson, J. Phys. Chem. Solids 11, 26 (1959).

${ }^{17}$ P.C. Hohenberg, Zh. Éksp. Teor. Fiz. 45, 1208 (1963) [Sov. Phys. JETP 18, 834 (1964)].

${ }^{18}$ A.A. Abrikosov and L.P. Gor'kov, Zh. Éksp. Teor. Fiz. 39, 1781 (1960) [Sov. Phys. JETP 12, 1243 (1961)]; Zh. Éksp. Teor. Fiz. 35, 1558 (1959) [Sov. Phys. JETP 8, 1090 (1959); 36, 319 (1959) [Sov. Phys. JETP 9, 220 (1959)].

${ }^{19}$ A.A. Abrikosov, L.P. Gor'kov and I.E. Dzyaloshinskii, Methods of Quantum Field Theory in Statistical Mechanics (Dover, New York, 1975).

${ }^{20}$ C.H. Choi and P. Muzikar, Phys. Rev. B 39, 11296 (1989).

${ }^{21}$ There is a misprint in Eq. (81) of Ref. 5: the factor $x_{c}$ in the second term of the expression for the coefficient $a$ was missed; $\pi^{2}$ in Eq. (78) was misprinted as $\pi^{3}$.
${ }^{22}$ C. Buzea and T. Yamashita, Supercond. Sci. Technol. 14, R115 (2001), Figs. 14, 15, and references therein; F. Bouquet, Y. Wang, I. Sheikin, P. Toulemonde, M. Eisterer, H.W. Weber, S. Lee, S. Tajima, and A. Junod, Physica C 385, 192 (2003).

${ }^{23}$ V.A. Moskalenko, M.E. Palistrant, and V.M. Vakalyuk, Usp. Fiz. Nauk 161, 155 (1991) [Sov. Phys. Usp. 34, 717 (1991)]; Eq. (2.11) and Eq. (4.9), Fig. 3 and Fig. 4.

${ }^{24}$ V.A. Moskalenko and M.E. Palistrant, Zh. Éksp. Teor. Fiz. 49, 770 (1965) [Sov. Phys. JETP 22, 536 (1966)].

${ }^{25}$ S.V. Shulga, S.-L. Drechsler, H. Eschrig, H. Rosner, and W.E. Pickett, cond-mat/0103154 (unpublished); H. Rosner, J.M. An, W. Ku, M.D. Johannes, R.T. Scaletter, W.E. Pickett, S.V. Shulga, S.-L. Drechsler, H. Eschrig, W. Weber, and A.G. Eguiluz, $M g B 2$ : Complex behavior from a simple compound, in Studies of High Temperature Superconductors, edited by A. Narlikar (Nova Science Publishers, New York, 2002), Vol. 38, p. 25; G. Fuchs, S.-L. Drechsler, A. Handstein, V. Narozhnyi, K. Nenkov, K.-H. Müller, and H. Rosner, in Studies of High Temperature Superconductors, edited by A. Narlikar (Nova Science Publishers, New York, 2002), Vol. 41, pp. 171-198.

${ }^{26}$ H. Choi, D. Roundy, H. Sun, M.L. Cohen, and S.G. Louie, Phys. Rev. B 66, 020513 (2002); Nature (London) 418758 (2002).

${ }^{27}$ D.A. Papaconstantopoulos and M.J. Mehl, Phys. Rev. B 64, 172510 (2002).

${ }^{28}$ A.A. Abrikosov, Physica C 214, 107 (1993).

${ }^{29}$ V.G. Kogan, Phys. Rev. B 66, 020509 (2002), Eq. (13).

${ }^{30}$ A.A. Abrikosov, Fundamentals of the Theory of Metals (North Holland, Amsterdam, 1988), Sec. 21, Eq. (21.5), Sec. 17.1 and references therein.

${ }^{31}$ A.A. Abrikosov, Physica C 244, 243 (1995).

${ }^{32}$ T. Takabatake and M. Ishikawa, Solid State Commun. 66, 413 (1988).

${ }^{33}$ H. Srikanth, S. Sridhar, D.A. Gaewsky, and M.B. Maple, Physica C 291, 235 (1997).

${ }^{34}$ G. Xiao, M.Z. Cieplak, J.Q. Xiao, and C.L. Chien, Phys. Rev. B 42, 8752 (1990).

${ }^{35}$ Y. Dalichaouch, M.C. de Andrade, D.A. Gajewski, R. Chau, P. Visani, and M.B. Maple, Phys. Rev. Lett. 75, 3938 (1995).

${ }^{36}$ S. Haas and K. Maki, Phys. Rev. B 65, 020502(R) (2001).

${ }^{37}$ T.M. Mishonov, E.S. Penev, and J.O. Indekeu, Phys. Rev. B 66, 066501(C) (2001).

${ }^{38}$ I.I. Mazin, O.K. Andersen, O. Jepsen, O.V. Dolgov, J. Kortus, A.A. Golubov, A.B. Kuz'menko, and D. van der Marel, Phys. Rev. Lett. 89, 107002 (2002), Fig. 1. 